\title{
Kleiner Aufwand, grosse Wirkung
}

\section{Matthias Scholer}

Wissenschaftsjournalist

\author{
Häusliche Gewalt, psychische Störungen und Substanzenabusus sind bekannte Ri- \\ sikofaktoren für eine Kindswohlgefährdung. In Holland hilft ein Screeningverfah- \\ ren auf Erwachsenennotfallstationen, Familien mit Risiken für eine Kindswohlge- \\ fährdung $\mathrm{zu}$ erkennen und Kindsmisshandlungen $\mathrm{zu}$ verhindern. Mit dem \\ Pilotprojekt SPEK wurde eine vergleichbares Verfahren auch in der Schweiz ge- \\ testet.
}

Letztes Jahr befragten die Mitarbeitenden der Notfallstationen des Kantonsspitals Bülach, des Spitals Limmattal und der Universitätsklinik Zürich während dreier Monate alle Patienten, die sie wegen Substanzenabusus, häuslicher Gewalt, schwerer psychischer Störung oder Suizidversuch betreuten, gezielt nach minderjährigen Kindern. Bestand eine Obhutspflicht, wurde mit dem Einverständnis der betreffenden Person eine Gefährdungsmeldung an die Kindes- und Erwachsenenschutzbehörde KESB gemacht. Verweigerte der Patient sein Einverständnis, konnte die Meldung erst nach einer Entbindung von der Schweigepflicht durch die Gesundheitsdirektion erfolgen. Während des Pilotversuchs meldeten die drei involvierten Spitäler insgesamt zwölf Patienten der KESB. Drei der insgesamt zwölf gemeldeten Personen bzw. deren Familien waren der KESB unbekannt.

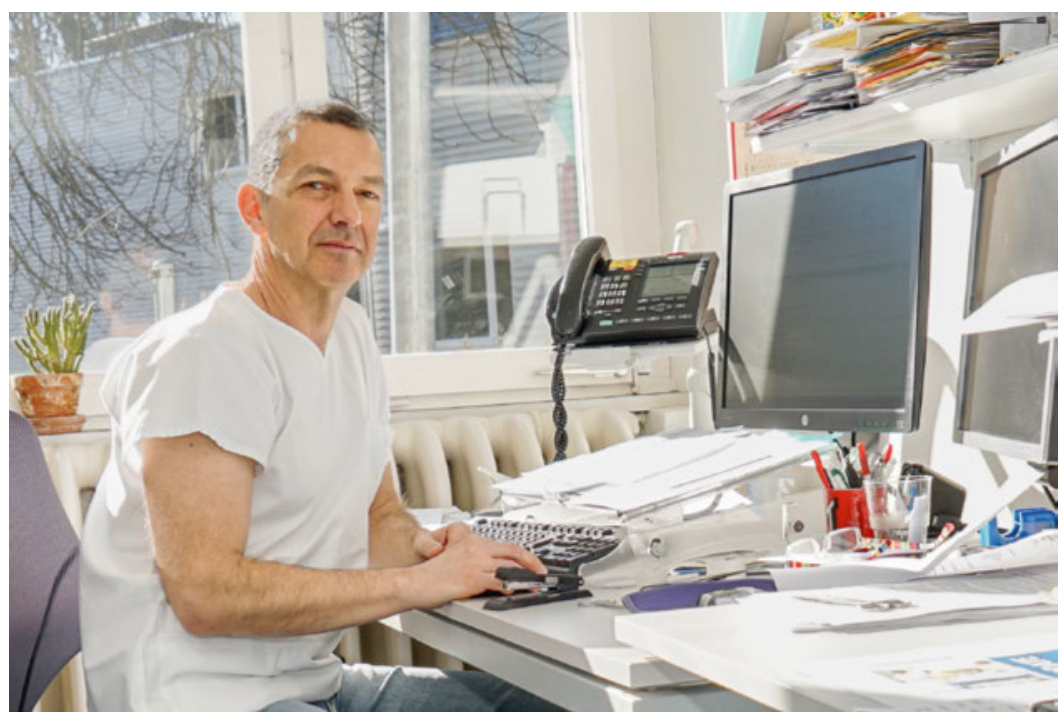

Georg Staubli leitet die Kinderschutzgruppe des Universitätskinderspitals Zürich.

\section{Herr Staubli, weshalb haben Sie diese Pilotstudie lanciert?}

Unserer Kinderschutzgruppe werden jedes Jahr rund 500 Kinder gemeldet, die vermutlich oder belegbar misshandelt wurden. Wir überlegten uns schon lange, welche Präventivmassnahmen helfen könnten, die seit Jahren gleichbleibend hohen Fallzahlen senken $\mathrm{zu}$ können. Als eine unserer Mitarbeiterinnen in ihrem Umfeld miterlebte, wie eine an Depressionen leidende Mutter einen Suizidversuch überlebte, danach ohne flankierende Hilfsangebote aus dem Spital entlassen wurde und sich kurz darauf das Leben nahm, entschlossen wir uns zu versuchen, ob das sogenannte The Hague Protocol [1] auch hierzulande Sinn machen würde.

\section{Können Sie uns dieses Protokoll kurz erklären?}

Es handelt sich dabei um ein in Holland angewendetes Screeningverfahren. Dabei werden Patienten, die wegen Substanzenabusus, häuslicher Gewalt, psychischer Störungen oder Suizidversuchen in die Notfallstationen eingeliefert wurden und eine Obhutspflicht für minderjährige Kinder innehaben, der Kinderschutzbehörde gemeldet. Diese klärt die familiären Verhältnisse ab und prüft, ob eine Kindswohlgefährdung vorliegt. Wird eine solche festgestellt, leitet die Behörde Unterstützungsmassnahmen ein.

\section{Zeigte das Verfahren in Holland Wirkung?}

Sehr sogar. Erstens stieg die Zahl gemeldeter Fälle merklich an. Zweitens konnte bei 91 Prozent der gemeldeten Fälle eine Kindswohlgefährdung festgestellt werden. Bei 90 Prozent dieser Fälle wurden anschliessend unterstützende Massnahmen eingeleitet. Dank dem Screening wurde somit eine hohe Anzahl gefährdeter Familien mit minderjährigen Kindern identifi- 
ziert, die den Behörden bislang unbekannt waren und somit auch keine Hilfestellungen erhielten.

Im Rahmen des Zürcher Pilotversuchs SPEK wurden während dreier Monate zwölf Patienten identifiziert, bei denen eine weitere Abklärung durch die KESB Sinn machte. Neun dieser zwölf Personen waren der KESB jedoch schon bekannt. Von den drei der KESB unbekannten Fälle wurde nur einer vertieft abgeklärt. Kann man daraus schliessen, dass die sozialen Strukturen in der Schweiz besser sind?

Um eine solche Aussage zu machen, sind die Zahlen unserer Pilotstudie schlicht zu klein. Aber auch wenn das Verhältnis tatsächlich nur 1:12 wäre, ändert sich aus meiner Sicht nichts an der Tatsache, dass es ein no-go ist, Eltern minderjähriger Kinder, die aufgrund von Substanzenabusus, häuslicher Gewalt oder psychischen Erkrankungen auf Notfallstationen eingeliefert wurden, ohne eine Hilfestellung insbesondere auch für die betroffenen Kinder einfach wieder zu entlassen. Die Weiterbetreuung könnte grundsätzlich auch vom Sozialdienst des Spitals übernommen werden. Das Problem ist jedoch, dass es in den meisten Fällen eine Behörde mit Befugnissen braucht. Deshalb bietet sich hierzulande die KESB als Meldestelle an.

Das Image der KESB ist durch Presseberichte, die von Fremdplatzierungen von Kindern gegen den Willen der Eltern berichten, ziemlich negativ ...

Drastische Massnahmen wie eine Fremdplatzierung sind in Tat und Wahrheit die Ausnahme. Dass viele Betroffene mit der Arbeit der KESB zufrieden sind, dringt kaum an die Öffentlichkeit. Tatsache ist, dass je früher die KESB Eltern in Krisensituationen unter die Arme greifen kann, desto niedrigschwelliger die Unterstützungsmassnahmen sind. Das Ziel muss doch sein zu intervenieren, solange die Situation noch rettbar ist.

\section{Aber besteht nicht die Gefahr, dass sich die betrof- fenen Personen fürchten, eine Notfallstation aufzu-} suchen, da sie dort denunziert werden können?

Dieses Bedenken hatten die Kollegen in Holland und in der Schweiz zu Beginn auch. Ich muss jedoch klar festhalten, dass es nicht darum geht, den Vater, der beispielsweise an einer Betriebsfeier über den Durst getrunken hat und danach eine Treppe hinuntergefallen ist, der KESB zu melden. Im Screeningprotokoll fehlt deshalb bewusst beispielsweise die Angabe einer Promillegrenze, bei deren Überschreitung eine Gefährdungsmeldung ausgelöst werden sollte. Jeder Fall muss individuell beurteilt werden. Die von uns angestrebten Gefährdungsmeldungen betreffen ausschliesslich Personen, die in medizinischen Ausnahmesituationen aufgefunden wurden und nicht mehr entscheidungsfähig sind. Von einer Denunzierung könnte man nur sprechen, wenn eine Notfallstation die Polizei einschaltet, um ein Strafverfahren zu eröffnen. Beim Screeningverfahren geht es jedoch primär darum, dem Personal der Notfallstationen die Möglichkeit einer Gefährdungsmeldung aufzuzeigen und den Behandelnden einen Denkanstoss zu geben, nicht nur den Patienten im Fokus zu haben, sondern auch die Auswirkungen auf dessen minderjährige Kinder zu bedenken.

Besteht denn keine Meldepflicht, wenn beispielsweise eine Mutter aufgrund häuslicher Gewalt ins Spital eingeliefert wird?

Interessanterweise unterliegt die Polizei einer Meldepflicht, wenn sie wegen häuslicher Gewalt gerufen wird und Kinder in diesem Haushalt anwesend sind. Diese Pflicht gibt es im Spitalbereich nicht. Das erstaunt, wenn man sich überlegt, dass wir uns nach einer Gewaltanwendung nicht mehr in einem Grau-, sondern bereits in einem Rotbereich befinden. In solchen Fällen sollte sich jede Ärztin, jeder Arzt verpflichtet fühlen, eine Kinderschutzbehörde zu beauftragen, einmal nach den Kindern in dem betroffenen Haushalt zu schauen.

\section{Das Pilotprojekt ist abgeschlossen. Wie soll es nun weitergehen?}

Wir brauchen mehr Zahlen. Deshalb sind wir daran, mit allen Erwachsenennotfallstationen im Kanton Zürich Kontakt aufzunehmen, um während mindestens eines Jahres das Protokoll in ihren Institutionen $\mathrm{zu}$ implementieren. Zusammen mit Vertretern der KESB klären wir die Entscheidungsträger der betroffenen Spitäler detailliert über die Abläufe auf und räumen allfällige Vorurteile und Missverständnisse aus. Ich bin überzeugt, dass niemand gegen ein Screening von Patienten auf Notfallstationen in Bezug auf eine Kindswohlgefährdung sein kann, wenn man den $\mathrm{Ge}$ danken dahinter verstanden hat.

\section{Bildnachweis \\ (c) Matthias Scholer}

Literatur

1 Diderich $\mathrm{H}$, et al. A new protocol for screening adults presenting with their own medical problems at the Emergency Department to identify children at high risk for maltreatment. Child Abuse \& Neglect. 2013;37(12):1122-31. doi: 10.1061/j.chiabu.2013.04.005

\section{Die Pilotstudie zum Nachlesen}

Die Pilotstudie kann unter saez.ch > Tour d'horizon > SPEK nachgelesen und heruntergeladen werden. 